\title{
Advanced Indoor Positioning Using Zigbee Wireless Technology
}

\author{
Marcin Uradzinski ${ }^{1} \cdot \mathrm{Hang}_{\mathrm{Guo}}^{2} \cdot \mathrm{Xiaokang}_{\mathrm{Liu}^{2}} \cdot$ \\ $\operatorname{Min} \mathbf{Y u}^{3}$
}

Published online: 16 August 2017

(C) The Author(s) 2017. This article is an open access publication

\begin{abstract}
The paper presents the results of the project which examines the level of accuracy that can be achieved in precision indoor positioning using a new improved Zigbee network fingerprint method. This method can provide more accurate positioning by filtering algorithm for improving the fingerprint Zigbee database accuracy. In our experiment the following two steps have been completed. Firstly, we filtered out the interference data generated in the fingerprint database source data acquisition process what improved the accuracy of establishing the fingerprint database. Next, the nearest algorithm, the weighted nearest algorithm and Bayesian algorithm were used to calculate pedestrian's location, and then the results are compared and analyzed. As a result, the average error with the improved fingerprint database is less than or equal to $0.81 \mathrm{~m}$ in a long distance range. The accuracy of the results got much better compared with the results without filtering. A newly developed ZigBee system by us can be applied to the location based services in a bigger space inside the buildings (distances up to $40 \mathrm{~m}$ ) or in the underground mines.
\end{abstract}

Keywords Wireless sensor networks - Zigbee technology $\cdot$ Indoor positioning · Navigation $\cdot$ Sensor integration

Marcin Uradzinski

marcin.uradzinski@uwm.edu.pl

1 Institute of Geodesy, University of Warmia and Mazury in Olsztyn, Oczapowskiego 1, 10-719, Olsztyn, Poland

2 Institute of Space Science and Technology, Nanchang University, P.O. Box 21-1-8, 202 West Beijing Rd, Nanchang, China

3 Department of Computer Science and Technology, Jiangxi Normal University, Nanchang 330022, China 


\section{Introduction}

As GPS is essential for outdoor navigation nowadays, locating a mobile user anytime anywhere is still a challenging task, especially in GPS degraded and denied environments such as urban canyons and indoors. Lately, some other positioning techniques have been developed such as wearable dead reckoning (DR) sensors, pseudolites, and MEMS to obtain a seamless indoor/outdoor positioning solution [1].

Wireless sensor networks, such as ZigBee, RFID, Wi-Fi, UWB etc., can provide RSSI (received signal strength indicator), which can be also used for positioning, especially indoor positioning, and therefore for location based services (LBS). For indoor environment, the fingerprint database method is more suitable for calculating the coordinates of a pedestrian location. As the development of modern science and technology, LBS and location-aware computing are increasingly important in the practical application. Currently, GPS positioning system is a mature positioning technology used widely, but microwave signals are easily absorbed, reflected by buildings, and attenuate seriously, so GPS positioning is not suitable for using in the indoor environment. In recent years, as the technology of internet of things develops rapidly, the establishment of wireless sensor networks will be more widely used in the indoor environment. This positioning method can use the signal strength indication between the reference nodes and positioning nodes, and design algorithms for positioning. In the wireless sensor networks, according to whether measuring the distance between the nodes in the positioning process, the positioning modes are divided into two categories which are range-based [2] and range-free positioning modes [3-7]. This paper describes the indoor positioning method based on RSSI fingerprint database, which is range-free.

The positioning method based on fingerprint database has to go through two stages: the offline fingerprint database establishment and the online positioning. In the offline fingerprint database establishment stage, we need to collect RSSI values of the reference nodes with the known coordinates, to establish the fingerprint database. In the online positioning stage, we compare the real-time RSSI values of the mobile nodes to the fingerprint database's information to calculate the locations of the mobile nodes. ZigBee system developed by us has been used for such indoor positioning and the accuracy reached $0.51 \mathrm{~m}$ (standard deviation) after improving a fingerprint database in a short distance range.

\section{Principle of Positioning}

The fingerprint database positioning is combined of two processes. The first stage establishes the fingerprint database and the second stage gets the coordinates of a mobile node in positioning area through RSSI values from each reference node. Handling this group RSSI values is shown in Fig. 1, so we can get the location of the mobile node in this area.

\subsection{Establishment of the Fingerprint Database}

The accuracy of the fingerprint database will directly affect the accuracy of the final positioning results. The high precision fingerprint database is the prerequisites of improving the positioning accuracy. The nearest data point for collecting RSSI value will always overlap when selecting the data point and collecting the RSSI values, so we choose 


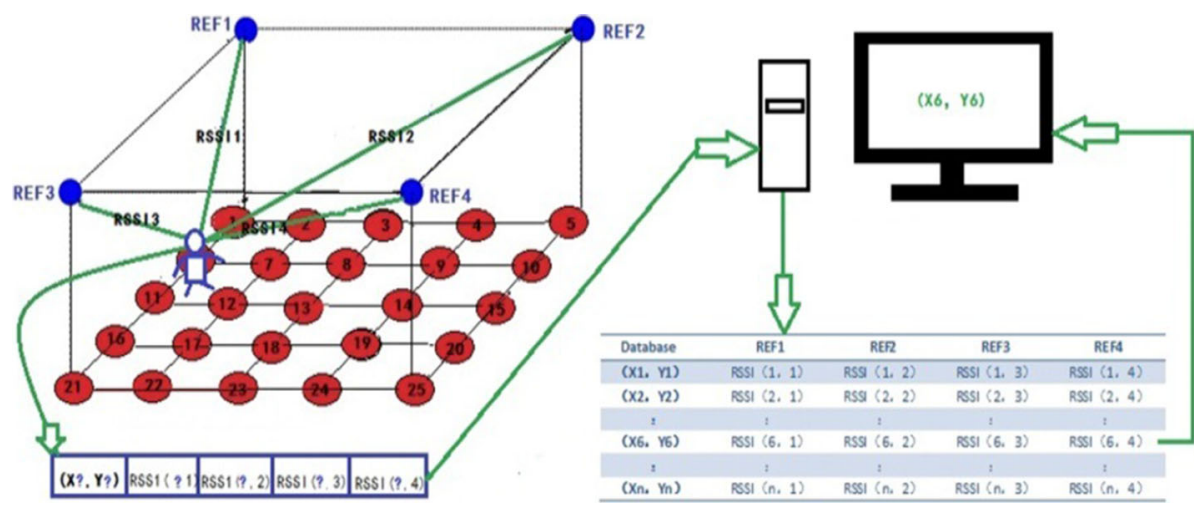

Fig. 1 The realization process of the fingerprint database positioning

the following sample data processing methods to improve the accuracy of the fingerprint database.

A group of data collected from data sample points is selected and analyzed. Define rssi (n)i be $\mathrm{n}$ groups of data of RSSI values from the $\mathrm{i}$-th reference node, namely: Rssi($\mathrm{n}) \mathrm{iR} \subseteq \mathrm{SSI}, \mathrm{n}$ is the data number of the group collected at this point, i represents RSSI values of the $\mathrm{i}$-th reference node.

In the data collection process, some groups of data may change abruptly because of interference. In order to prevent the harmful data from incorporating into the fingerprint database, an upper and lower threshold binding with experiments is set. The average RSSI value of the collection point which receive from the i-th reference node can be expressed as:

$$
\mathrm{u}_{\mathrm{i}}=\sum_{1}^{\mathrm{N}} \operatorname{rssi}(\mathrm{n})_{\mathrm{i}} / \mathrm{N}
$$

$\theta$ as the change factors, i.e.:

$$
\begin{aligned}
& \operatorname{rssi}(\mathrm{n})_{\mathrm{i}} \in\left[\mathrm{u}_{\mathrm{i}}-\theta, \mathrm{u}_{\mathrm{i}}+\theta\right] \\
& \operatorname{rssi}(\mathrm{n})_{\mathrm{i}} \notin\left[\mathrm{u}_{\mathrm{i}}-\theta, \mathrm{u}_{\mathrm{i}}+\theta\right]
\end{aligned}
$$

When Eq. (2) makes, it indicates that there is no abnormal of data in data collection process. When Eq. (3) makes, it indicates that abnormal occur, it should be removed and replaced by its average $u_{i}$.

After obtaining data from each data collection point and eliminating the influence of the abnormal, we also found problem in the data collection. It's that frequency of occurrence of data far away from the data center point at both ends is very low. The emergence of these data is the overlapping of the nearest sampling points, which is incorporated directly into meta date of the fingerprint database and will affect the accuracy of the database. For this case, we can eliminate the influence with a data domain filtering method. The specific approach expresses as follows: Set domain zone of the data collected as $\{\operatorname{rssi}(\mathrm{n}) \mathrm{il}[\mathrm{min}$, $\max ])\}$ in which min and max represent the maximum value and the minimum value of the group of RSSI values collected. Mid is the center value, the central offset is $\xi$, i.e.: the effective data domain of this group of data is $\{\operatorname{rssi}(n) i \mid[\min , \max ])\}$, we select weights of 
data which appear in [min, Mid- $\xi$ ) and (Mid $+\xi$, max] area in accordance with the order of distance from the center value, and then weight and average, naturally form a new group of data, finally, we weight and average this new group of data and the data in valid data domain in accordance with number of occurrences totally. Calculating as follows:

$$
\begin{gathered}
\operatorname{rssi}(\mathrm{n})_{\varepsilon 0}=\frac{\varepsilon}{\theta} \operatorname{rssi}(\mathrm{n})_{\varepsilon}+\frac{\theta-\varepsilon}{\theta} \operatorname{rssi}(\mathrm{n})_{\varepsilon+1} \\
\operatorname{rssi}(\mathrm{n})_{\varepsilon 0-1}=\frac{\mathrm{k}}{\mathrm{k}+\varphi} \operatorname{rssi}(\mathrm{n})_{\varepsilon-1}+\frac{\varphi}{\mathrm{k}+\varphi} \operatorname{rssi}(\mathrm{n})_{\varepsilon 0} \\
K_{0}=k+
\end{gathered}
$$

$\operatorname{rssi}(n)_{\varepsilon 0}$ denote the new RSSI values which are the weighted averages of the $\varepsilon t h$ RSSI value and the $(\varepsilon+1)$ th RSSI value. $\theta$ is the number of groups of different RSSI values of the data center point on both sides. The farther the distance is from the center the bigger $\theta$ is. $\varepsilon$ is the number of sequence number of these groups.

The $\operatorname{rssi}(n)_{\varepsilon 0-1}$ denotes the new RSSI values, which are the weighted averages of the $(\varepsilon-1)$ th RSSI values in accordance with the number of each group of data. $\varphi$ represents the total number of data of $\varepsilon, \varepsilon+1$ groups, $\mathrm{k}$ represents the number of data of original, $\mathrm{K} 0$ denotes the new the number of data of $\varepsilon-1$ groups. After operation of Eqs. (5), (6), and (7), the edges of the value in the source data are weighted and merged, then the data is optimized.

What is shown in Fig. 2 is the fingerprint database establishment process. First the certain number of data sampling points is selected in the positioning area, then fingerprint database source data is collected, and then the filter processing on the source data to eliminate "Invalid" data is performed, finally the fingerprint database of location is established.

\subsection{Fingerprint Database Positioning Algorithm}

Three locating algorithms to estimate the positions of the user are introduced as follows:

1. The nearest algorithm

The nearest positioning algorithm [8,9] used a series of sampling points selected in positioning area during the offline stage for positioning. These points are denoted as

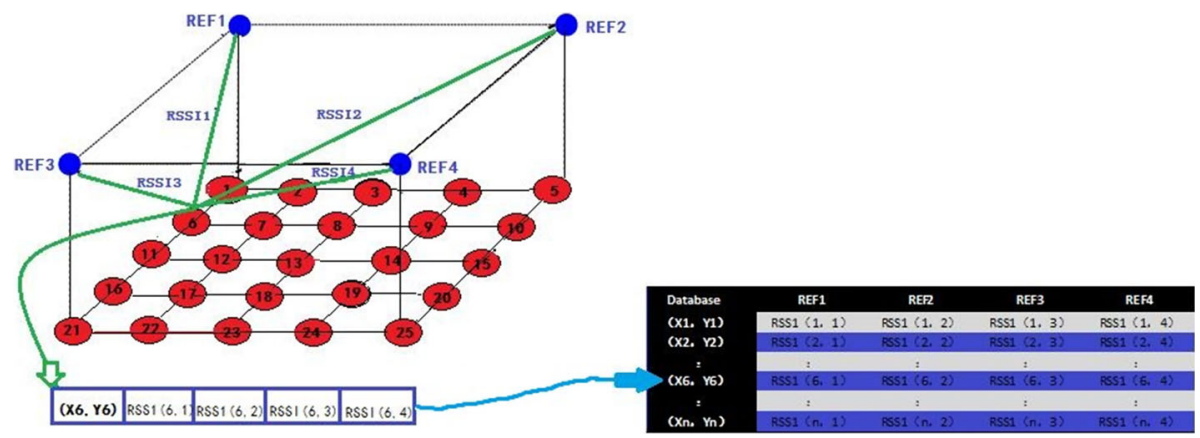

Fig. 2 The process of establishing a fingerprint database 
$\mathrm{Fi} \in\{\mathrm{F} 1, \mathrm{~F} 2, \ldots, \mathrm{Fn}\}$. When the moving nodes get into the positioning area in the online positioning stage, we can get the RSSI location fingerprint sampling values on a real-time basis denoted as $\mathrm{S}$. It contains signal strength values received from $\mathrm{m}$ reference nodes, it is $\mathrm{S}=(\mathrm{S} 1, \mathrm{~S} 2, \ldots, \mathrm{Sm})$. Through comparing the distance $\mathrm{S}$ and Fi, we can know that the point corresponding to the most nearest position fingerprint is the positioning point. Then we choose the Euclidean distance to measure the proximity of the two. Calculated as follows:

$$
\mathrm{Dn}=\sqrt{\sum_{\mathrm{i}=1}^{\mathrm{m}}(\mathrm{Si}-\mathrm{RSSI})^{2}}
$$

The minimum Euclidean distance is the estimated position of the positioning node $\mathrm{L}=\min \mathrm{D}(\mathrm{S}, \mathrm{Fi})$.

\section{The weighted nearest algorithm}

Taking into account the complex indoor environment, measurement error is large. So we use the weighting factor $1 / \mathrm{Dj}$ to weight and average the coordinate values of the location fingerprint information points among the smaller K Euclidean distance. Closer the distance between the fingerprint information points, the larger the weight [10]. Its weight value can be expressed as

$$
\mathrm{W}_{\mathrm{j}}=\frac{1 / \mathrm{Dj}}{\sum_{\mathrm{j}=1}^{\mathrm{k}} 1 / \mathrm{Dj}}
$$

So, the user's location can be calculated using the following formula:

$$
\mathrm{L}=\sum_{\mathrm{j}=1}^{\mathrm{k}} \mathrm{W}_{\mathrm{j}} * \mathrm{~L}_{\mathrm{j}}
$$

$\mathrm{Wj}$ is the weight value of the $\mathrm{j}$-th fingerprint information point, and $\mathrm{Lj}$ is the coordinate of the $\mathrm{j}$-th fingerprint information point.

\section{Bayesian algorithm}

Bayesian algorithm [9] obtain the position fingerprint feature of the sampling points in the off-line stage, it is different from the RSSI values of the nearest algorithm, the location fingerprint characteristic is the probability distribution of all the received RSSI values of beacon nodes received from the sampling point. We can obtain the distribution of RSSI values of each beacon node. A RSSI location fingerprint sample can be denoted as S. It contains signal strength values received from $m$ reference nodes, it is $S=(S 1, S 2, \ldots, S m)$. Bayesian algorithm is to obtain the real-time posterior probability of each position where the RSSI location fingerprint sample $\mathrm{S}$ in the pointing area, this can be denoted as $\mathrm{P}(\mathrm{Lil} \mathrm{S})$. According to Bayes theory, posterior probability can be further deduced as the following equation:

$$
\mathrm{P}(\mathrm{Li} \mid \mathrm{S})=\frac{\mathrm{P}(\mathrm{S} \mid \mathrm{Li}) * \mathrm{P}(\mathrm{Li})}{\mathrm{P}(\mathrm{S})}=\frac{\mathrm{P}(\mathrm{S} \mid \mathrm{Li}) * \mathrm{P}(\mathrm{Li})}{\sum_{\mathrm{k} \in \mathrm{L}} \mathrm{P}(\mathrm{S} \mid \mathrm{Lk}) * \mathrm{P}(\mathrm{Lk})}
$$

In the above equation, $\mathrm{P}(\mathrm{S} \mid \mathrm{Li})$ is called the conditional probability of the real-time RSSI location fingerprint sample $\mathrm{S}$ in the case of some locations known. $\mathrm{P}(\mathrm{Li})$ is called priori probability in location area of Li. Since users may be at any location in the positioning 
area, we can think that $\mathrm{P}(\mathrm{Li})$ obeys the uniform distribution. Because the received signal strength of each beacon node is independent and irrelevant, so $\mathrm{P}(\mathrm{S} \mid \mathrm{Li})$ calculation can be expressed as:

$$
\mathrm{P}(\mathrm{S} \mid \mathrm{Li})=\mathrm{P}(\mathrm{S} \mid 1 \mathrm{~L} 1) * \mathrm{P}(\mathrm{S} 2 \mid \mathrm{L} 2) * \cdots * \mathrm{P}(\mathrm{Sm} \mid \mathrm{Li})
$$

We can use Gaussian probability distribution expressing the distribution of RSSI values at the certain position, then:

$$
\mathrm{P}(\mathrm{S} \mid \mathrm{Li})=\frac{1}{\sqrt{2 \pi * \sigma}} \exp \left[-\frac{\mathrm{s}-\mathrm{u}^{2}}{2 \sigma^{2}}\right]
$$

where $\mu$ and $\delta$ respectively represent the mean and standard deviation of the signal strength. We use the maximum posteriori probability to estimate the location of the users, that is $\mathrm{L}=\max \mathrm{P}(\mathrm{LilS})$.

\section{Experimental Results and Analysis}

\subsection{The Deployment of Experimental Environment}

The selection rule of the density of collection points of the fingerprint database data: the more intensive the selection of the fingerprint data, the higher positioning accuracy [11]. When using the cc2430 system of Texas Instrument and selecting a different interval data collection points in the test area, we find that if the intervals between the collection points are less than $0.5 \mathrm{~m}$, RSSI values from the four references to nodes nearby several nearest data sampling points are the same. So in the experiment, we select $1.6 \mathrm{~m}$ as the sampling point interval to establish a fingerprint database.

In the experimental area $(42.5 \mathrm{~m} \times 4.96 \mathrm{~m}$, we select 108 data sampling points shown in Fig. 3), the white points denote the selected fingerprint data sampling points, the interval between the points is $1.6 \mathrm{~m}$. Each point collected 150 groups of data, and then we extract RSSI values from the sampling data of each reference node, calculate its mean and variance and establish the positioning fingerprint database of off-line stage. In order to eliminate the anthropogenic influences of operating devices in the beginning and the end phases of the sampling of each data sample point, we remove 25 groups of data which is in the front and rear of the 150 collected fingerprint information point data respectively, and each sampling point keep only 100 groups of data.

We first removed 25 groups of data which is in the front and the rear of the 150 collected fingerprint information point data respectively, the remaining 100 groups of data, and there were some jump points. If these points are included in the database, the accuracy of the fingerprint database will be affected.

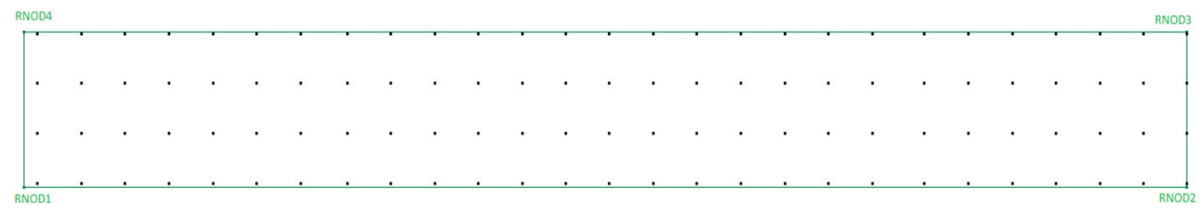

Fig. 3 The layout of reference nodes and the distribution of the fingerprint data points 
After processing average threshold values and selecting valid data domains to above sampling data, abnormal data was filtered by the averaging threshold method, and the offset data within a certain range was optimized by the method of selecting valid data domains.

After processing the collected data in the method of selecting valid data domains, the fingerprint data is centralized and the edge scatter data reduces.

\subsection{Positioning Results}

After establishing the fingerprint database and choosing actual route, we calculate the real time positions in the location area. In Fig. 4, the red line denotes actual route (with modeled 1-second positions for comparison), and blue line stands for Zigbee positioning results according to the fingerprint database.

Based on previous experiments we know that no matter what algorithm is used to calculate the position, the positioning accuracy of the filtered sampling data is higher than the accuracy without filtering. In this experiment we also used the nearest algorithm and Bayesian algorithm, but the best results in this case were obtained using the weighted nearest algorithm what is shown in Fig. 5 and Table 1.

All results show that the standard deviation with the improved fingerprint database is less than or equal to $0.51 \mathrm{~m}$ in a long distance range.

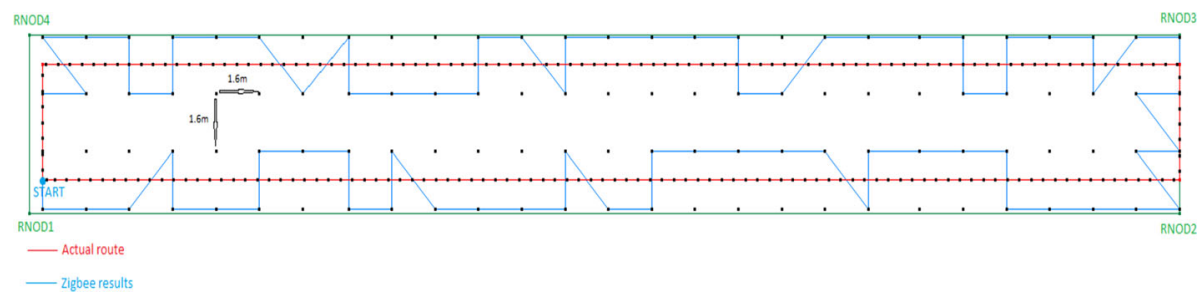

Fig. 4 Zigbee positioning results

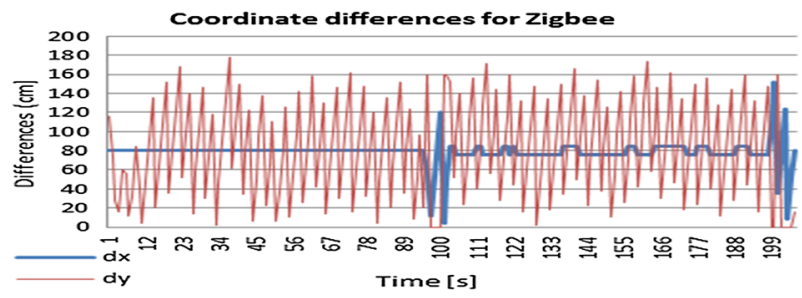

Fig. 5 Zigbee positioning results (weighted nearest algorithm)

Table 1 Summary of Zigbee positioning errors (weighted nearest algorithm)

\begin{tabular}{lrr}
\hline & $\mathrm{dx}$ & $\mathrm{dy}$ \\
\hline Min $(\mathrm{cm})$ & 4 & 0 \\
Max (cm) & 152 & 178 \\
Average $(\mathrm{cm})$ & 78 & 81 \\
SD $(\mathrm{cm})$ & 13 & 51 \\
\hline
\end{tabular}




\section{Conclusions}

The fingerprint database positioning can provide more accurate positioning results, and the accuracy of establishing fingerprint database will affect the accuracy of indoor positioning. In this paper, we put forward a new method about the average threshold and the effective data domain filtering method to optimize the fingerprint database of ZigBee. This new ZigBee chip based on Texas Instrument cc2530 system has been developed by our navigation group at Nanchang University, Nanchang, China, in the year 2013. Conducted indoor experiment proved that the distance achieved by this hardware has been extended to nearing $40 \mathrm{~m}$ without decreasing the positioning accuracy. The weighted nearest algorithm was chosen and used to calculate user's location, and then the results were compared and analyzed. As a result, the positioning accuracy can be improved and achieved within $0.51 \mathrm{~m}$ (standard deviation) in the optimal case, so the system can be applied in a bigger space inside the buildings, underground mines or in the other location based services.

Acknowledgements The paper was supported by the project of the Agreement on Scientific and Technological Cooperation between the Government of the Republic of Poland and the Government of the People's Republic of China-"A Research of Key Technology of MEMS/IMU/Radio Frequency Indoor Positioning".

Open Access This article is distributed under the terms of the Creative Commons Attribution 4.0 International License (http://creativecommons.org/licenses/by/4.0/), which permits unrestricted use, distribution, and reproduction in any medium, provided you give appropriate credit to the original author(s) and the source, provide a link to the Creative Commons license, and indicate if changes were made.

\section{References}

1. Guo, H., Uradzinski, M., Yin, H., \& Min, Y. (2015). Indoor positioning based on foot-mounted IMU. Bulletin of the Polish Academy of Sciences-Technical Sciences, 63(3), 629-634.

2. Yan, J., Tiberius, C. C. J. M., Janssen, G. J. M., Teunissen, P. J. G., \& Bellusci, G. (2013). Review of range-based positioning algorithms. IEEE Aerospace and Electronic Systems Magazine, 28(8), 2-27.

3. Maddio, S., Passafiume, M., Cidronali, A., \& Manes, G. (2015). A distributed positioning system based on a predictive fingerprinting method enabling sub-metric precision in IEEE 802.11 networks. IEEE Transactions on Microwave Theory and Techniques, 63(12), 4567-4580.

4. Weike, C., Wenfeng, L., Heng, S., \& Bing, Y. (2006). Weighted centroid localization algorithm based on RSSI for wireless sensor networks. Journal of Wuhan University of Technology (Transportation Science \& Engineering), 30(2), 265-268.

5. Lott, M., \& Forkel, I. (2001). A multi-wall-and-floor model for indoor radio propagation. In IEEE vehicular technology conference 53rd (Vol. 1, pp. 464-468).

6. Morales, J., Akopian, D., \& Agaian, S. (2016). Mitigating anomalous measurements for indoor wireless local area network positioning. IET Radar, Sonar and Navigation, 10(7), 1220-1227.

7. Patwari, N., Hero, A. O., Perkins, M., Correal, N. S., \& O’Dea, R. J. (2003). Relative location estimation in wireless sensor networks. IEEE Transactions on Signal Processing, 51(8), 2137-2147.

8. Wang, H., Zhao, Z., Hu, J., Qu, Z., \& Feng, H. (2016). Study on improvement of fingerprint matching algorithm in wireless LAN based indoor positioning system. In 2016 17th IEEE/ACIS international conference on software engineering, artificial intelligence, networking and parallel/distributed computing (SNPD) (pp. 275-280), Shanghai.

9. Zhang, X., Guo, H., Wu, H., \& Uradzinski, M. (2014). New indoor fingerprint database positioning algorithm using nonlinear interpolation and particle filter. Geodesy and Cartography, 63(2), 219-233.

10. Patwari, N., Hero, A. O., \& Costa, J. A. (2007). Learning sensor location from signal strength and connectivity. Advances in Information Security, 30(Part I), 57-81.

11. Li, B., Wang, Y., Lee, H. K., Dempster, A., \& Rizos, C. (2005). Method for yielding a database of location fingerprints in WLAN. IEEE Proceedings Communications, 152(5), 580-586. 

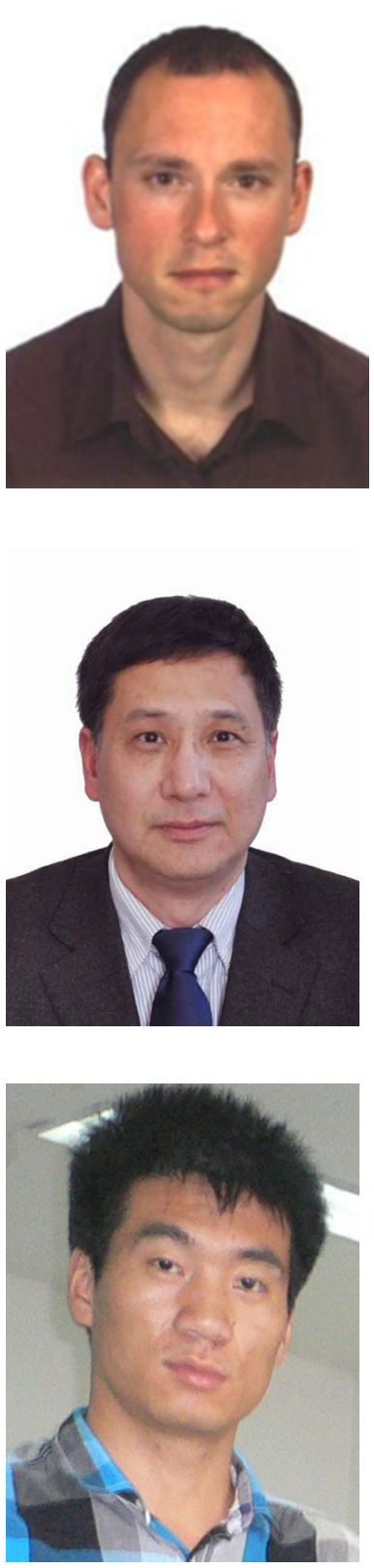

Marcin Uradzinski got his Ph.D. in the Faculty of Geodesy and Land Managment with emphasis on satellite positioning and navigation from University of Warmia and Mazury, Poland. He is currently a visiting Professor at Nanchang University. His interests include satellite positiong, multi-sensor integrated navigation and indoor radio navigation system.

Hang Guo got his Ph.D. in Geomatics and Geodesy with emphasis on navigation from Wuhan University, China. Currently, he is a Professor of Academy of Space Technology at Nanchang University, and member of ION. His interests include indoor positioning, multi-sensor integrated navigation system, and GNSS MET.

Xiaokang Liu is a graduate student at Nanchang University. His interests include indoor radio positioning system, hardware and software development. 


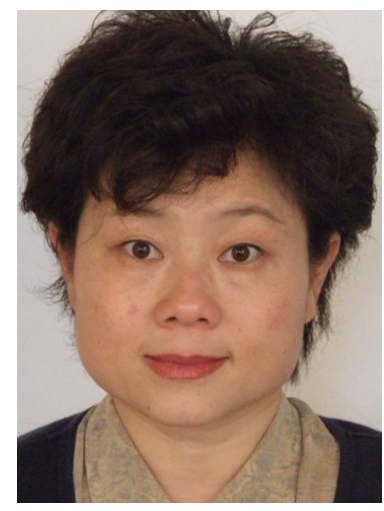

Min Yu is a Professor at Jiangxi Normal University, Nanchang, major in Electronic Engineering and Communication, computer, and interested in Indoor positioning and sensor networking. 